Full Length Article

\title{
Large-scale quantification of human osteocyte lacunar morphological biomarkers as assessed by ultra-high-resolution desktop micro-computed tomography
}

\author{
Elliott Goff ${ }^{\mathrm{a}}$, Federica Buccino ${ }^{\mathrm{a}}$, Chiara Bregoli ${ }^{\mathrm{a}}$, Jonathan P. McKinley ${ }^{\mathrm{a}, \mathrm{b}}$, Basil Aeppli ${ }^{\mathrm{a}}$, \\ Robert R. Recker ${ }^{c}$, Elizabeth Shane ${ }^{\mathrm{d}}$, Adi Cohen ${ }^{\mathrm{d}}$, Gisela Kuhn ${ }^{\mathrm{a}}$, Ralph Müller ${ }^{\mathrm{a}, *}$ \\ ${ }^{\text {a } I n s t i t u t e ~ f o r ~ B i o m e c h a n i c s, ~ E T H ~ Z u r i c h, ~ Z u r i c h, ~ S w i t z e r l a n d ~}$ \\ ${ }^{\mathrm{b}}$ Department of Mechanical Engineering, University of California Berkeley, Berkeley, CA, USA \\ ${ }^{\mathrm{c}}$ Department of Medicine, Creighton University Medical Center, Omaha, NE, USA \\ ${ }^{\mathrm{d}}$ Department of Medicine, Columbia University Vagelos College of Physicians \& Surgeons, New York, NY, USA
}

\section{A R T I C L E I N F O}

\section{Keywords:}

Micro-CT

Lacuna

Morphometry

Large-scale

Human

Osteocyte

\begin{abstract}
A B S T R A C T
Ultra-high-resolution imaging of the osteocyte lacuno-canalicular network (LCN) three-dimensionally (3D) in a high-throughput fashion has greatly improved the morphological knowledge about the constituent structures positioning them as potential biomarkers. Technologies such as serial focused ion beam/scanning electron microscopy (FIB/SEM) and confocal scanning laser microscopy (CLSM) can image in extremely high resolution, yet only capture a small number of lacunae. Synchrotron radiation computed tomography (SR-CT) can image with both high resolution and high throughput but has a limited availability. Desktop micro-computed tomography (micro-CT) provides an attractive balance: high-throughput imaging on the micron level without the restrictions of SR-CT availability.

In this study, accuracy, reproducibility, and sensitivity of large-scale quantification of human osteocyte lacunar morphometries were assessed by ultra-high-resolution desktop micro-computed tomography. For this purpose, thirty-one transiliac human bone biopsies containing trabecular and cortical regions were imaged using ultra-highresolution desktop micro-CT at a nominal isotropic voxel resolution of $1.2 \mu \mathrm{m}$. The resulting 3D images were segmented, component labeled, and the following morphometric parameters of 7.71 million lacunae were measured: Lacunar number (Lc.N), density (Lc.N/BV), porosity (Lc.TV/BV), volume (Lc.V), surface area (Lc.S), surface area to volume ratio (Lc.S/Lc.V), stretch (Lc.St), oblateness (Lc.Ob), sphericity (Lc.Sr), equancy (Lc.Eq), and angle (Lc. $\theta$ ).

Accuracy was quantified by comparing automated lacunar identification to manual identification. Mean true positive rate (TPR), false positive rate (FPR), and false negative rate (FNR) were $89.0 \%, 3.4 \%$, and $11.0 \%$, respectively. Regarding the reproducibility of lacunar morphometry from repeated measurements, precision errors were low $(0.2-3.0 \%)$ and intraclass correlation coefficients were high (0.960-0.999). Significant differences between cortical and trabecular regions $(p<0.001)$ existed for Lc.N/BV, Lc.TV/BV, local lacunar surface area $(<$ Lc.S $>$ ), and local lacunar volume $(<$ Lc.V $>)$, all of which demonstrate the sensitivity of the method and are possible biomarker candidates. This study provides the foundation required for future large-scale morphometric studies using ultra-high-resolution desktop micro-CT and high-throughput analysis of millions of osteocyte lacunae in human bone samples.
\end{abstract}

\footnotetext{
Abbreviations: LCN, lacuno-canalicular network; FIB/SEM, focused ion beam/scanning electron microscopy; CLSM, confocal laser scanning microscopy; SR-CT,

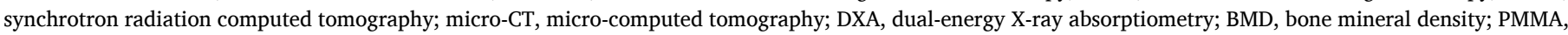

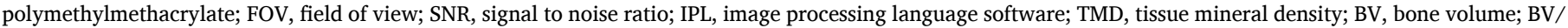

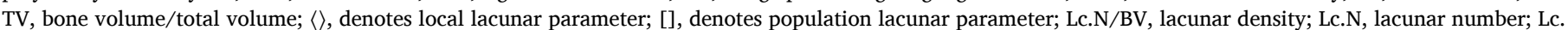

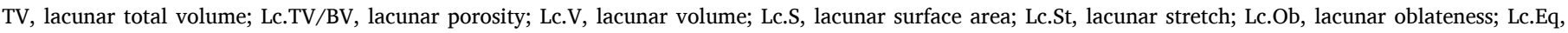

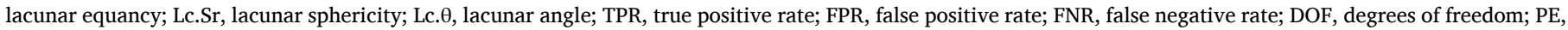

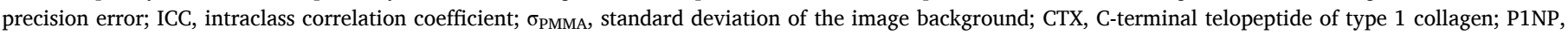
Procollagen type I N-terminal propeptide.

* Corresponding author at: Institute for Biomechanics, ETH Zurich, Leopold-Ruzicka-Weg 4, 8093 Zurich, Switzerland.

E-mail address: ram@ethz.ch (R. Müller).
} 


\section{Introduction}

Bone as an organ provides humans with the necessary structural support to sustain locomotion and dynamic movement in daily life. The organ is uniquely capable of adapting its structure to meet the mechanical demands that are placed upon it [1]. This adaptation of bone has been described by Roux as bone (re)modeling [2]. Central to this process are the osteocytes: the most abundant bone cell type, embedded deeply within the bone matrix, and each ensconced within individual compartments called lacunae [2,3]. Woven together by a large number of dendrites that extend from each cell, the lacuno-canalicular network (LCN) is one of the most intricately connected networks in the human body, and the scale is comparable with the network of neurons in the human brain [4]. Compelling studies over the last thirty years have revealed and emphasized the functional importance of the cells and processes within the LCN to sense mechanical signals, to transduce them into chemical signals, and to orchestrate the bone (re)modeling process through guided bone formation and bone resorption [5-10].

After cell death, the fossilized lacuna remains intact, allowing the lacuna's three-dimensional (3D) geometry to be extracted via several imaging techniques at the sub-micrometer resolution. Today, serial focused ion beam/scanning electron microscopy (FIB/SEM) possesses the highest spatial resolution in the nanometer range. Yet, while this technology allows for features like individual dendritic processes to be resolved, the depth range is a major limitation, and only a few dozen lacunae in the tissue can be captured simultaneously [11-14]. Other researchers have implemented confocal laser scanning microscopy (CLSM) to investigate lacunar geometry in mice $[15,16]$ and in clinical bone biopsies [17], but again the shallow tissue depth that can be explored is a limitation and hence only small subsections of bone consisting of a few dozen to a few hundred lacunae can be measured with CLSM. Both FIB/SEM and CLSM suffer from a lack of scalability since the time required for a study with more than a few hundred lacunae makes the technologies impractical for any type of large-scale lacunar analysis. Alternatively, several groups have used high-resolution x-ray-based approaches such as synchrotron radiation computed tomography (SRCT). Several studies, Mader et al. [18] in particular, have been successful in separating the porous lacunae from the surrounding matrix in a highthroughput fashion in complete intact mouse femurs [18-24]. However, SR-CT is an imaging tool that requires access to a beamline facility, of which only a few in the world exist, and hence the availability is limited for most researchers due to timing restrictions. A fourth imaging tool, conventional X-ray based ultra-high-resolution desktop micro-computed tomography (micro-CT), provides a reasonable balance between CLSM and SR-CT. Ultra-high-resolution desktop micro-CT allows for the extraction of millions of lacunae from complete bone biopsies without the need to request approval for limited time slots or experienced personnel at beamline facilities. Furthermore, desktop micro-CT is an established and validated technology that has been implemented for laboratory-based bone research for several decades [25-28]. Therefore, it is necessary that a technique be developed for large-scale, highthroughput imaging of osteocyte lacunar networks in clinical bone biopsies.

Equally as important as the 3D images acquired are the individual structures that are extracted from these images as well as the accurate, reproducible, and sensitive quantification of their morphometry. Specifically, with ultra-high-resolution osteocyte imaging, it is imperative that the lacunar morphometric parameters are well defined and measured accordingly. Great strides have been made towards the standardization of these metrics in recent years, and several studies have explored different basic measures such as lacunar density, shape, and orientation [18,20,21,29-34]. Mader et al. have most thoroughly described and validated both simple and abstract lacunar morphometric parameters, and hence this study follows their naming convention and mathematical definitions [18]. The combination of well-defined lacunar morphometric parameters and a validated imaging and analysis methodology allow for the emergence of biomarkers. These morphometric biomarkers have the potential to be used to differentiate between diseased and healthy bone, old and young bone, or even the region of bone within the body.

This study aims to provide researchers with a reliable method of large-scale lacunar imaging, accurate automated identification, and measurements of each resulting 3D lacunar structure using a technology that is widely accessible - ultra-high-resolution desktop micro-CT. Furthermore, we demonstrate the power of such a high-throughput analysis by measuring the morphometric parameters of millions of osteocyte lacunae in human bone samples, using the previously validated 3D lacunar metrics of Mader et al. [18]. The rigor of the method is confirmed by accurate lacunar identification, a standard precision study [35], and the sensitive detection of differences between cortical and trabecular regions. The demonstrated validation highlights the value of the imaging method, and we believe this study will provide a rigorous foundation for future large-scale lacunar investigations.

\section{Methods}

\subsection{Human bone biopsy preparation}

Thirty-one transiliac bone biopsy samples from premenopausal women already described in previous studies by Cohen et al. [36,37] were used for this study. Biopsy samples had been obtained in women, aged 18-48, recruited as a reference population for studies of bone structure and metabolism in premenopausal women. Reference population subjects were required to have normal areal spine, hip, and forearm BMD by dual-energy X-ray absorptiometry (DXA; Z score $\geq$ -1.0 at all sites), no history of adult low trauma fracture, and no historical or biochemical evidence of diseases or conditions known to affect skeletal integrity [36,37]. All subjects provided written informed consent; studies had been approved by the institutional review boards of all participating institutions.

A hemicylinder fraction of each biopsy core, containing both cortical and trabecular regions, was embedded in individual polymethylmethacrylate (PMMA) disks with a $25 \mathrm{~mm}$ diameter. Subsections of each sample were prepared to fit in the desktop micro-CT scanner, which limits the diameter to a $4.0 \mathrm{~mm}$ field of view (FOV) in the ultrahigh-resolution mode. Hence, each PMMA embedded core was cut three times parallel to the longitudinal axis of the biopsy using a circular diamond blade (SCAN-DIA Minicut 40, SCAN-DIA GmbH \& Co. KG, Germany) and custom-made mounts for sample fixation. This produced a rectangular block, which had the XY target dimensions of $4.25 \pm 0.1$ $\mathrm{mm}$ with the $\mathrm{Z}$ dimension depending on the original placement in the PMMA disk and ranging from 20 to $25 \mathrm{~mm}$. Each block was then turned on a conventional lathe (Schaublin 102, Bevilard, Switzerland) to create a final cylinder of $3.8 \pm 0.05 \mathrm{~mm}$ diameter and a length ranging between 10 and $15 \mathrm{~mm}$. The biopsy cylinder was then tightly fit into a custom sample holder as seen in Fig. 1 to minimize motion artifacts and to maximize the volume scannable within the $4.0 \mathrm{~mm}$ diameter FOV.

\subsection{Image acquisition}

Biopsy subsamples were imaged with a $\mu$ CT50 (Scanco Medical AG, Brüttisellen, Switzerland), operated with a $0.5 \mathrm{~mm}$ aluminum filter, 72 $\mu \mathrm{A}$ current, $4 \mathrm{~W}$ power, $55 \mathrm{kVp}$ energy, $1.5 \mathrm{~s}$ integration time, level 6 data averaging, and with a total of 1500 projections. Images were reconstructed at a nominal isotropic voxel resolution of $1.2 \mu \mathrm{m}$ with an anti-ring level 8 to minimize center ring artifacts using the manufacturer's scanner software. Each image consisted of a cylindrical volume equal to the full diameter of the sample $(3.8 \pm 0.05 \mathrm{~mm})$ and the height of one scan stack ( 909 slices $=1.09 \mathrm{~mm}$ ). The protocol for each sample consisted of three scans: 1 . Prescan to warm the sample in the scanner gantry in an effort to reduce motion artifacts caused by thermal effects $(1 \mathrm{~h}) ; 2$. Cortical region scan starting from the lowest point on the 
centerline of the sample and scanning up one stack (10h); and 3. Trabecular region scan stack in the middle of the biopsy equidistant between both cortical walls $(10 \mathrm{~h})$. A visual example of this scanning protocol and resulting images can be seen in Fig. 1.

To determine the optimal beam energy, three samples of trabecular bone were scanned at three beam energies: 55, 70, and $90 \mathrm{kVp}$. These samples used for beam energy optimization originated from a separate study [38]; however, they were also human bone biopsies from the iliac crest and were considered to be comparable with our biopsy group. We aimed to maximize the image signal-to-noise ratio (SNR) just as previous studies relating to other anatomical bone sites had done [39]. The linear attenuation coefficient (raw signal) was measured for ten twodimensional sub-regions $\left(\sim 0.25 \mathrm{~mm}^{2}\right)$ at every beam energy in three samples for 90 regions in total. SNR was then calculated by adapting the Firbank equation to account for two distinct materials as described in Eq. (1) where $\mu$ is the average coefficient of linear attenuation of bone and the background (PMMA) and $\sigma$ is the standard deviation of the background [40].

$\mathrm{SNR}=0.655 \frac{\mu_{\text {bone }}-\mu_{\mathrm{PMMA}}}{\sigma_{\mathrm{PMMA}}}$

\subsection{Image preprocessing}

Preprocessing of each image consisted of a constrained 3D Gaussian low pass filter $(\sigma=0.8$, support $=1)$ to reduce noise and was applied using IPL (Scanco Medical AG, Brüttisellen, Switzerland). Segmentation of lacunar structures was performed by inverting the image after applying a threshold that was individualized for each image volume, which was necessary due to the large variation in tissue mineral density (TMD) distributions between samples at this resolution. The lacunar threshold was determined by fitting a Gaussian distribution to each sample's raw TMD distribution data and calculating the first critical point $\left(\mathrm{g}^{\prime}\right)$ of the fitted distribution using a custom Python script (3.7.1, Python Software Foundation, Delaware, USA). Bone volume (BV) was determined by fixing a threshold of $520 \mathrm{mg} \mathrm{HA} / \mathrm{ccm}$, applying to all samples, performing a closing operation, and calculating the resulting BV using IPL software (Scanco Medical AG, Brüttisellen, Switzerland) (Fig. 2).

All objects smaller than $50 \mu \mathrm{m}^{3}$ and larger than $2000 \mu \mathrm{m}^{3}$ were removed as to reflect the range of human lacunar volumes reported in previous histological studies [41]. The lower bound filters out noise structures while the upper bound excludes larger porosities like Haversian and Volkmann canals. Similar volumetric ranges have also been implemented in previous studies [42,43]. Several microcracks, blood vessels, and image ring artifacts escaped the volumetric filter, yet all exhibited similar thin structures with a high object-elongation value. Therefore, these were excluded by removing all objects with an elongation above a threshold (Lc.St $>0.85$ ). All objects sharing a border with the image edge were also removed to exclude partially cutoff objects.

\subsection{Image morphometry}

The lacunar morphometries were calculated with a custom Python script, which first component labeled all lacunar objects, applied a surface mesh to each, and then measured basic individual parameters. Lacunar density (Lc.N/BV) was calculated by normalizing the number of lacunae (Lc.N) to the bone volume (BV) and lacunar porosity (Lc.TV/ $\mathrm{BV}$ ) by dividing the total volume of all lacunae by the respective BV. Local parameters (denoted with \langle\rangle and first defined by Stauber et al. [44]) were each normalized to the sample Lc.N while population-based parameters (denoted with []) were not normalized. Individual lacunar volume (Lc.V), lacunar surface area (Lc.S), and the eigen vectors were determined from the object-specific mesh. This mesh was calculated by performing a triangulation of the surface voxels of the object using Lewiner marching cubes (3.7.1, Python Software Foundation, scikitimage library, Delaware, USA). The eigen vectors were then used to quantify more complex parameters including lacunar stretch (Lc.St) and lacunar oblateness (Lc.Ob), which were first defined by Mader et al. [18]. Lacunar equancy (Lc.Eq) was the ratio between the smallest and largest eigen vectors (E3/E1) [21,45] while lacunar sphericity (Lc.Sr) related the lacunar object to a sphere via the ratio between Lc.S and Lc.V [43]. Lacunar angle (Lc. $\theta$ ) was measured in degrees and ranged between 0 and $180^{\circ}$ in relation to an arbitrarily created unit vector that was held consistent between images.

\subsection{Validation}

\subsubsection{Accuracy of automated lacunar identification}

To evaluate the accuracy of the automated threshold approach, lacunae from five sample sub-volumes consisting of roughly 200 lacunae per region were manually identified by a trained observer by moving

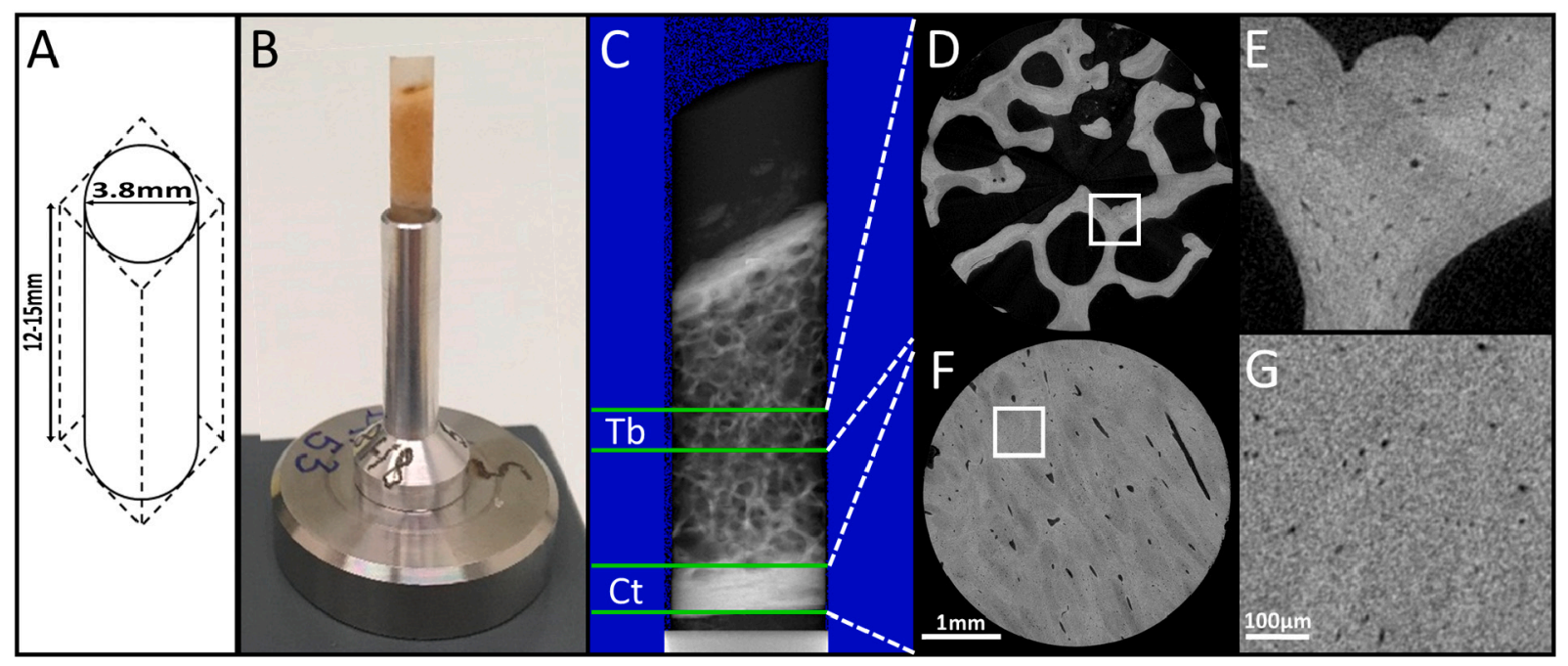

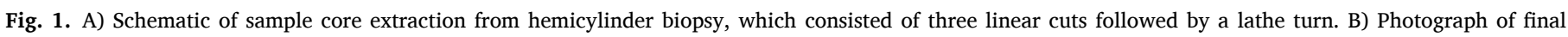

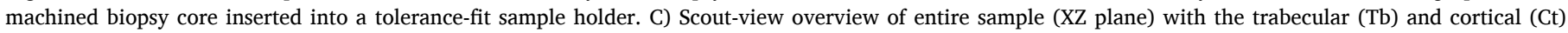

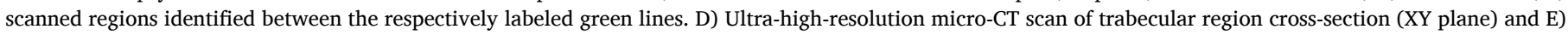

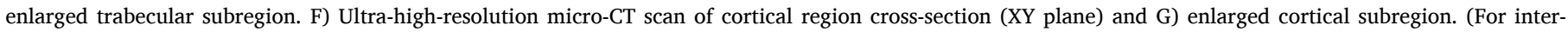
pretation of the references to color in this figure legend, the reader is referred to the web version of this article.) 

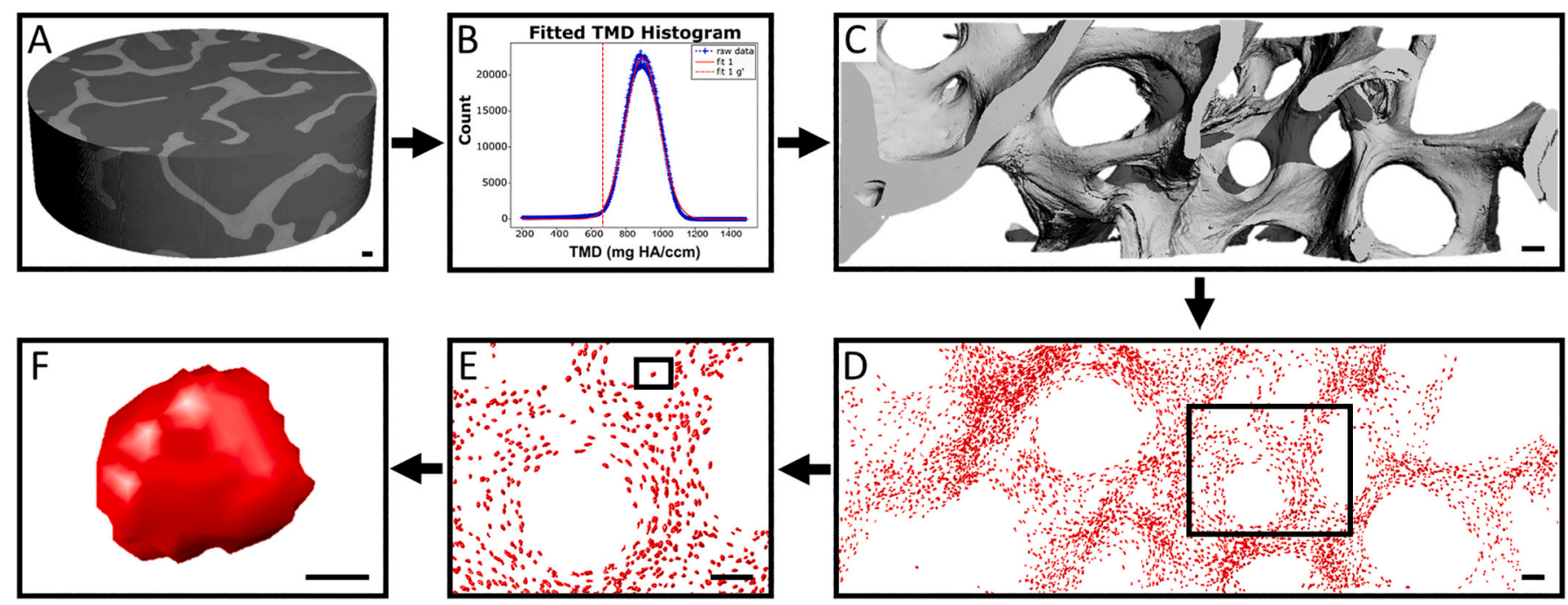

Fig. 2. Visual overview of large-scale lacunar segmentation. A) Gaussian filtered, unsegmented, complete micro-CT image stack. B) Sample specific histogram of tissue mineral density (TMD) values fitted by a Gaussian function. A unique lacunar threshold is chosen for each sample at the first critical point of the respective fit by calculating the maximum of the first derivative $\left(\mathrm{g}^{\prime}\right)$. C) Lacunar threshold calculated with (B) applied to (A). D) Image (C) inverted and component labeled to identify lacunae. E) Enlarged subregion group of lacunae. F) Single visualized lacuna. Scale bar lengths: A-E) $100 \mu \mathrm{m}$; F) $10 \mu \mathrm{m}$.

through stacks of slices and then compared with the automatically identified lacunae at the corresponding sample-specific $\mathrm{g}^{\prime}$ threshold to calculate true positive, false positive, and false negative rates (TPR, FPR, FNR). All objects were verified in 3D as depicted in Fig. 3 and falsely classified objects were reclassified when appropriate. The geometry of false negatives was evaluated in $2 \mathrm{D}$ while the respective center voxel position of the objects could be evaluated in 3D in relation to successfully segmented true positive and false positive objects. Objects which were characterized as false positives typically were jagged, irregular in shape, and did not generally resemble lacunae in both 2D and 3D.

\subsubsection{Reproducibility of repeated measurements}

In accordance with repeated measurements literature that recom- mends a sufficient number of degrees of freedom (DOF) to produce an upper confidence limit of the precision error that is $40 \%$ greater than the mean precision error [35], six samples were measured five times and repositioned between each measurement for a total of 20 DOF. Reproducibility of repeated measurements was evaluated by calculating the precision error ( $\mathrm{PE}_{\% \mathrm{CV}}$ ) using Eqs. (2)-(3) where $\mathrm{m}$ is the quantity of subjects, SD represents the standard deviation of $\mathrm{m}$ repeated measures on subject $\mathrm{j}$, and $\mathrm{CV}$ is the coefficient of variation. The intraclass correlation coefficient (ICC) for the measured lacunar indices was calculated using Eq. (4) where $\mathrm{n}$ represents the number of repetitions and $\mathrm{F}_{0}$ represents the ratio of the residual within-subject mean squares and the between-subject mean squares.

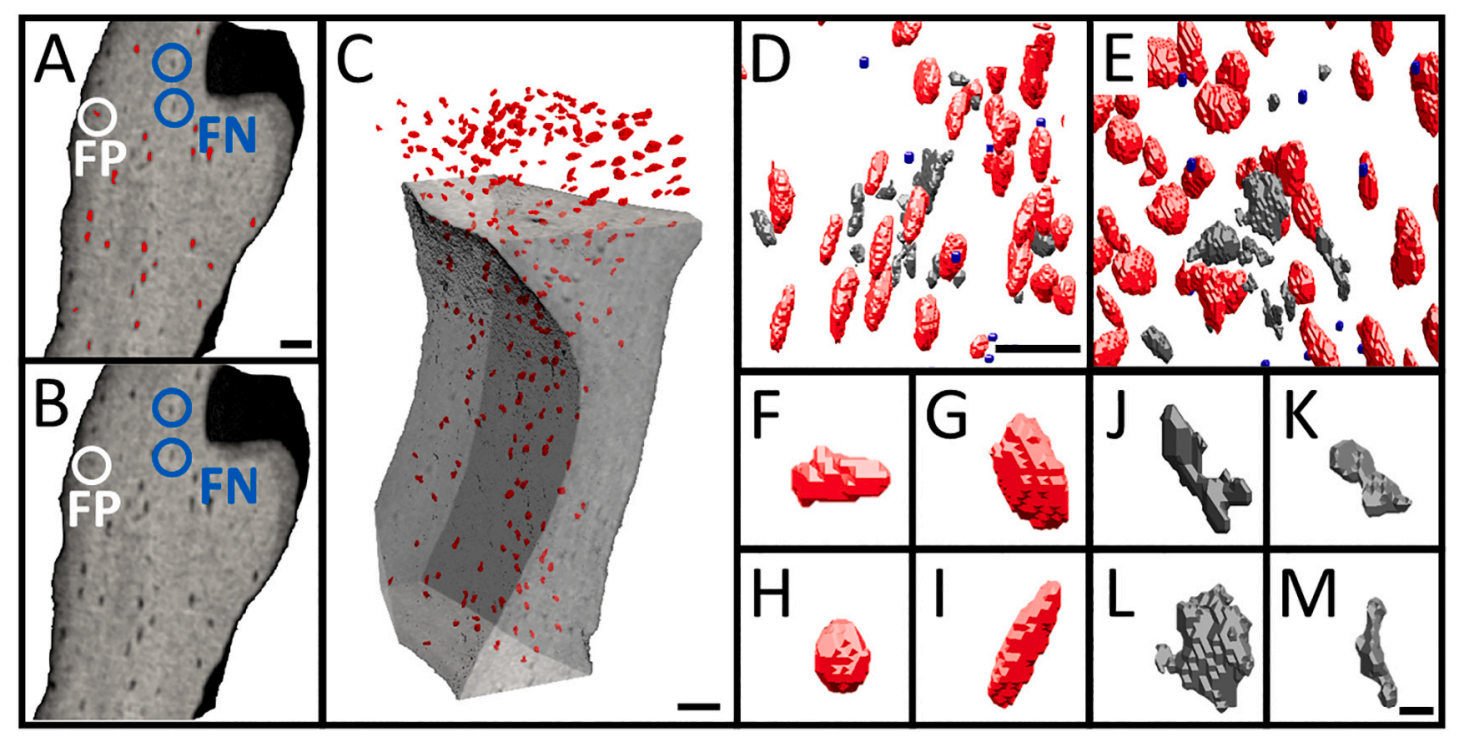

Fig. 3. Manual vs. automatic lacunar identification. A) 3D cutplane of trabecular subregion with automatically segmented objects highlighted in red. Examples of false negatives (FN) circled in blue, false positives (FP) circled in white. B) Same cutplane as (A) used for visual comparison regarding classification of automatically segmented objects, showing same FP and FN as in (A). C) 3D orthographic projection of (A \& B) with automatically segmented objects in red. D \& E) 3D visualization comparing manual and automatic lacunar identification. Red objects = true positives (TP); objects identified as lacunae both manually and automatically. Gray objects = false positives (FP); objects identified as lacunae automatically but rejected manually. Blue spheres = false negatives (FN); center voxel of objects manually identified as lacunae but rejected by the automatic method. F-I) TP examples. J-M) FP examples. Scalebar A-E) $=50 \mu \mathrm{m} ; \mathrm{F}-\mathrm{M})=10 \mu \mathrm{m}$. (For interpretation of the references to color in this figure legend, the reader is referred to the web version of this article.) 
$P E_{S D}=\sqrt{\sum_{j=1}^{m} \frac{S D_{j}^{2}}{m}}$

$P E_{\% C V}=\sqrt{\sum_{j=1}^{m} \frac{\% C V_{j}^{2}}{m}}$

$I C C=\frac{F_{0}-1}{F_{0}+(n-1)}$

\subsubsection{Sensitivity to biological differences}

To assess the method's sensitivity to biological differences, lacunar morphometric parameters from cortical and trabecular regions were compared as there are known physiological differences between the distribution and shape of lacunae in these regions in humans [43,46]. Because each individual biopsy contained both cortical and trabecular regions, it was possible to compare lacunae both within samples and between samples. This sensitivity analysis was inspired by the previous work of Nebuloni et al. [47] and we extend the definition here to be the ability to detect biological differences, in this case regional lacunar differences, which have been previously identified [43,46].

\subsection{Statistical analysis}

A paired Student's $t$-test with the necessary Bonferroni correction was performed with respect to energy-dependent imaging parameters in Table 1 . With respect to the precision test, the $95 \%$ confidence interval was calculated for each morphometric parameter using a chi-squared distribution to gain an understanding of certainty with respect to the PE and ICC reported values in Table 3. Creating two-parameter plots of the data presented in Table 3 is another way to evaluate the reproducibility of the imaging method by means of clustering as is depicted in Fig. 5. A paired Student's $t$-test was performed to evaluate regional differences between lacunar parameters that were normalized to tissue indices $(p<0.001)$. Population-based parameters were not normally distributed following a Kolmogorov-Smirnov test, and so a nonparametric Mann-Whitney $U$ test was performed to evaluate population differences $(p<0.001)$. The inter-quartile ranges and medians were computed for each morphometric parameter.

\section{Results}

\subsection{Image acquisition}

Images captured with a $55 \mathrm{kVp}$ beam energy exhibited a significantly higher SNR when compared to images obtained with a beam energy of $90 \mathrm{kVp}(p<0.005)$. The SNR measured with the $70 \mathrm{kVp}$ beam energy fell in between the high and low beam energy values and did not differ significantly from the other energies.

As shown in Table 1, both contrast and standard deviation of the background (PMMA in this case) were inversely proportional to the beam energy. Specifically, the inverse proportionality was approximately linear with contrast while $\sigma_{\mathrm{PMMA}}$ was quadratic (Fig. 4), both

Table 1

Energy dependency of imaging parameters. Energy level $55 \mathrm{kVp}$ used as baseline and paired $t$-test with the necessary Bonferroni correction used to test for significant differences between energy levels in the three following categories: contrast, standard deviation of the image background ( $\left.\sigma_{\mathrm{PMMA}}\right)$, and signal-tonoise ratio (SNR). Significantly different $(p<0.005)$ from baseline denoted with (*) and from $70 \mathrm{kVp}$ with (\#).

\begin{tabular}{llll}
\hline Energy $(\mathrm{kVp})$ & Contrast $(\mathrm{n}=30)$ & $\sigma_{\text {PMMA }}(\mathrm{n}=30)$ & SNR $(\mathrm{n}=30)$ \\
\hline 55 & $1.84 \pm 0.46$ & $0.14 \pm 0.02$ & $8.34 \pm 2.20$ \\
70 & $1.33 \pm 0.33^{*}$ & $0.11 \pm 0.01^{*}$ & $7.65 \pm 1.88$ \\
90 & $0.92 \pm 0.23^{*} \#$ & $0.09 \pm 0.01^{*} \#$ & $6.67 \pm 1.60^{*}$ \\
\hline
\end{tabular}

important for the computation of SNR as given in Eq. (1). As a ratio of both noise and the standard deviation of the image background (Eq. (1)), SNR was less dramatically affected by increasing beam energy. We therefore set the beam energy to $55 \mathrm{kVp}$ for all scans in the study because this setting produced the highest quality images with the highest contrast at acceptable noise levels.

\subsection{Validation}

\subsubsection{Accuracy of automated lacunar identification:}

Automatically identified objects were compared with the manually identified objects to evaluate the true positive rate (TPR), false positive rate (FPR), and false negative rate (FNR) measures as seen in Table 2. Objects identified automatically in each sample revealed a strong agreement with the lacunae counted manually as is evident by the high TPR and low FPR and low FNR. Roughly 200 lacunae were present in each sample subregion with sample 1 exhibiting the highest TPR (93.9\%) and lowest FNR (6.1\%). Sample 4 had the lowest FPR (0.5\%) while sample 3 the lowest TPR (77.3\%). The final lacunar identification accuracy measure was calculated as the mean of the five samples and was computed to be $89.0 \%$ TPR, 3.4\% FPR, and $11.0 \%$ FNR.

\subsubsection{Reproducibility of repeated measurements:}

Repeated measurement reproducibility is crucial for validation and was quantified for lacunar indices. Across all lacunar morphometric parameters, precision errors were very low (below 3\%) and the ICC were very high (above 0.980 ), which indicate that the measurements of these lacunar parameters are extremely reproducible. The 95\% confidence interval range for precision errors was between $0.67 \%$ and $3.68 \%$ while the range for ICC values were between 0.883 and 1.000 , which indicates extremely low measurement variability and high reproducibility.

Clustering of the individual repeated measurements indicates reproducibility of lacunar morphometry and is especially apparent in Fig. 5A-B. These parameters also exhibited a very high correlation, which illustrated that as bone volume increases so will the number of lacunae and the total lacunar volume. The clustering in Fig. 5C-D was not nearly as evident across all six samples due to the fact that $\langle\mathrm{Lc} . \mathrm{V}\rangle$ and $\langle\mathrm{Lc}$.St $\rangle$ were more difficult to reproduce. As BV/TV increases in Fig. 5C-D, measured variability decreases between the first and second cluster and then remains approximately constant. The strong correlation of Lc.N $\left(\mathrm{R}^{2}=0.99\right)$ and Lc.TV $\left(\mathrm{R}^{2}=0.98\right)$ with BV/TV position them as potential lacunar biomarker candidates. $\langle\mathrm{Lc} . \mathrm{V}\rangle$ and $\langle\mathrm{Lc} . \mathrm{St}\rangle$ were more independent of BV/TV and no correlation was found.

\subsubsection{Sensitivity to biological differences}

Cortical and trabecular bone regions were measured and compared to evaluate the sensitivity of the method to biological differences. More bone was present, and consequently, more lacunae were present in cortical bone when compared to trabecular bone. Measured tissue values such as $\mathrm{BV}$, and $\mathrm{BV} / \mathrm{TV}$ in trabecular regions were consistently lower than cortical regions as expected since trabecular bone is sparse and cortical bone is compact. Regarding the global morphometries, which were normally distributed, Lc.N/BV median value in trabecular bone was nearly half of what it was observed to be in cortical bone $(16,611$ vs. $26,429, p<0.001$ ). Similarly, the median value of Lc.TV/BV in trabecular bone was also nearly half of what it was in cortical bone ( $0.30 \%$ vs. $0.58 \%, p<0.001)$, again indicating that cortical bone has a significantly higher lacunar porosity than trabecular bone. Furthermore, we report in Table 4 that the normalized local parameters $\langle$ Lc.V $\rangle$ and $\langle$ Lc.S $\rangle$ are significantly greater $(p<0.001)$ in cortical bone $(\langle\mathrm{Lc} . \mathrm{V}\rangle=$ $223 \mu \mathrm{m}^{3} ;\langle$ Lc.S $\left.\rangle=233 \mu \mathrm{m}^{2}\right)$ than in trabecular bone $\left(\langle\mathrm{Lc} . \mathrm{V}\rangle=178 \mu \mathrm{m}^{3}\right.$; $\langle\mathrm{Lc} . \mathrm{S}\rangle=194 \mu \mathrm{m}^{2}$ ). Most population-based morphometric parameters were not normally distributed, and consequently we reported the median values as well as the interquartile range for all indices to provide a sense of the distribution of each parameter in Table 4.

Population-based lacunar parameters were not normally distributed. 


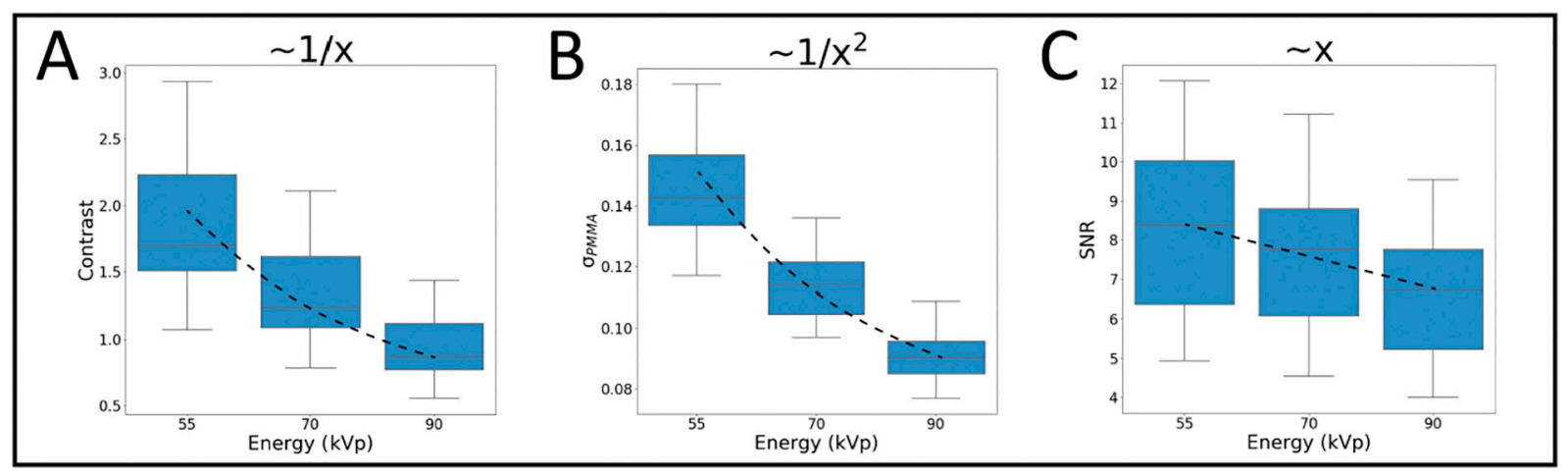

Fig. 4. Beam physics relationships. Ten subregions measured at each energy (30 measurements in total). A) Contrast exhibits an inverse relationship with beam energy. B) Standard deviation of the background $\left(\sigma_{\mathrm{PMMA}}\right)$ has an approximate quadratic relationship with beam energy. C) Signal-to-noise ratio is linearly related to beam energy as expected since it is defined as the contrast divided by the $\sigma_{\mathrm{PMMA}}$.

Table 2

Quantification of accuracy measures and their corresponding rates obtained via comparison between manual and automatic lacunar identification methods. $\mathrm{TPR}=$ true positive rate; $\mathrm{FPR}=$ false positive rate; $\mathrm{FNR}=$ false negative rate.

\begin{tabular}{llrlllc}
\hline Sample & TP & FP & FN & TPR (\%) & FPR (\%) & FNR (\%) \\
\hline 1 & 155 & 9 & 10 & 93.9 & 5.5 & 6.1 \\
2 & 182 & 11 & 14 & 92.9 & 5.7 & 7.1 \\
3 & 133 & 3 & 39 & 77.3 & 2.2 & 22.7 \\
4 & 202 & 1 & 16 & 92.7 & 0.5 & 7.3 \\
5 & 205 & 7 & 27 & 88.4 & 3.3 & 11.6 \\
Mean & 175 & 6 & 21 & 89.0 & 3.4 & 11.0 \\
\hline
\end{tabular}

The interquartile ranges between regions were similar, yet a nonparametric Mann-Whitney U test revealed significant differences between cortical and trabecular regions $(p<0.001)$. The measure of sphericity ([Lc.Sr]) was approximately 0.75 for both regions of bone, indicating similarities between the measured ellipsoids and an idealized sphere. The additional parameters [Lc.Eq], [Lc.St] and [Lc.Ob] allow for the ellipsoidal lacunar shape to be described in more detail, and the median reported values suggest that these are indeed ellipsoidal structures in both trabecular and cortical regions. The range of [Lc.Ob] in trabecular bone was slightly higher than in cortical bone.

Fig. 6 depicts selected global, local, and population-based morphometries from both cortical and trabecular regions. When normalized to the analyzed tissue volume, both the lacunar density and porosity were significantly different between cortical and trabecular regions (Fig. 6A-B) across all 31 samples, further supporting their potential as biomarkers. Local morphometries ( $\langle$ Lc.V $\rangle$ \& $\langle$ Lc.S $\rangle$ ) were also significantly different between the two regions (Fig. 6C-D), yet not as clearly separated as the global morphometries. Population-based morphometries (Fig. 6E-F) included all lacunar observations across all 31 samples: 1.14 million lacunae in trabecular bone and 6.57 million lacunae in cortical bone. The shape indices [Lc.St] and [Lc.Sr] were chosen to compare between regions and were also significantly different. A visual comparison is presented in Fig. $6 \mathrm{G}-\mathrm{H}$ between the samples containing the median Lc.N/BV values from Fig. 6A, further illustrating the differences between anatomical regions.

\section{Discussion}

Detailed examination of the LCN on a large scale demands a highresolution 3D-imaging methodology that is accurate, reproducible, and sensitive. Lacunae must be identified accurately and morphometric indices measured repeatably. Therefore, it is paramount that researchers select a validated imaging methodology for the large-scale assessment of osteocyte lacunar morphometry. High-resolution desktop micro-CT is an ideal technology for large-scale investigation of the lacunar network due to its wide accessibility. Micro-CT has been employed for decades as a standard technology for bone tissue morphometry with nominal voxel resolutions in the range of 10-40 $\mu \mathrm{m}$ [25-28]. However, this technology has evolved in recent years, and with it, the ability to image higher resolutions on the order of $1 \mu \mathrm{m}$ [48]. Hence, the development of validated image acquisition, processing, and analysis tools to image lacunar morphometry is a logical progression of the science as well as being crucial to understanding the biological impact of the LCN on bone at other hierarchical levels.

Identifying the lacunar structures to be analyzed required a

Table 3

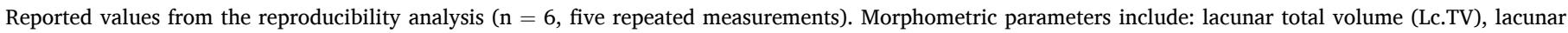

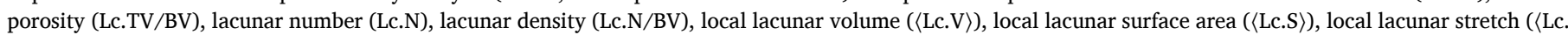

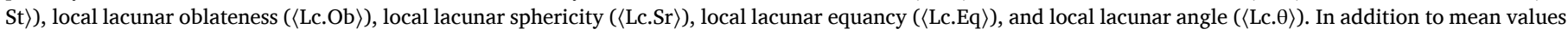

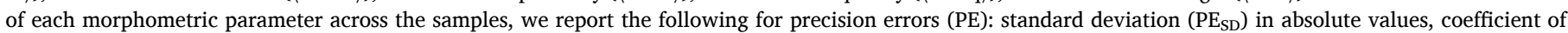

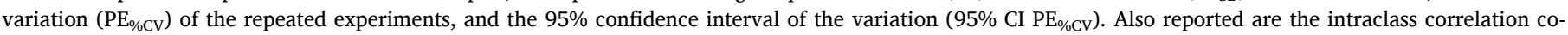
efficients (ICC) and the $95 \%$ confidence interval of the ICC for each respective morphometric parameter.

\begin{tabular}{|c|c|c|c|c|c|c|}
\hline Morphometric parameter & Mean & $\mathrm{PE}_{\mathrm{SD}}$ & $\mathrm{PE}_{\% \mathrm{CV}}$ & 95\% CI PE\%CV & ICC & 95\% CI ICC \\
\hline Lc.TV $\left(1000 * \mu \mathrm{m}^{3}\right)$ & 2119.3 & 40.2 & $2.77 \%$ & $2.11-4.05 \%$ & 0.997 & $0.992-1.000$ \\
\hline Lc.TV/BV (\%) & 0.5 & 0.009 & $2.52 \%$ & $1.91-3.68 \%$ & 0.989 & $0.966-0.998$ \\
\hline Lc.N (1000) & 10.03 & 0.2 & $1.86 \%$ & $1.41-2.72 \%$ & 0.999 & $0.996-1.000$ \\
\hline Lc.N/BV $\left(1000 / \mathrm{mm}^{3}\right)$ & 22.09 & 0.3 & $1.48 \%$ & $1.12-2.16 \%$ & 0.984 & $0.952-0.997$ \\
\hline$\langle\mathrm{Lc} . \mathrm{V}\rangle\left(\mu \mathrm{m}^{3}\right)$ & 210.5 & 2.5 & $1.33 \%$ & $1.01-1.95 \%$ & 0.991 & $0.970-0.999$ \\
\hline$\langle$ Lc.S $\rangle\left(\mu \mathrm{m}^{2}\right)$ & 207.5 & 1.7 & $0.88 \%$ & $0.67-1.29 \%$ & 0.990 & $0.968-0.998$ \\
\hline$\langle$ Lc.St $\rangle(1)$ & 0.6 & 0.001 & $0.17 \%$ & $0.13-0.25 \%$ & 0.989 & $0.963-0.998$ \\
\hline$\langle\mathrm{Lc} . \mathrm{Ob}\rangle(1)$ & -0.4 & 0.006 & $1.59 \%$ & $1.21-2.32 \%$ & 0.960 & $0.883-0.994$ \\
\hline$\langle\mathrm{Lc} . \mathrm{Sr}\rangle(1)$ & 0.8 & 0.002 & $0.24 \%$ & $0.18-0.35 \%$ & 0.938 & $0.816-0.990$ \\
\hline$\langle$ Lc.Eq $\rangle(1)$ & 0.3 & 0.001 & $0.43 \%$ & $0.33-0.63 \%$ & 0.994 & $0.980-0.999$ \\
\hline$\langle$ Lc. $\theta\rangle\left(^{\circ}\right)$ & 113.8 & 0.8 & $0.66 \%$ & $0.50-0.97 \%$ & 0.980 & $0.938-0.997$ \\
\hline
\end{tabular}


Table 4

Large-scale lacunar morphometric parameters $(n=6.57$ million for cortical and $\mathrm{n}=1.14$ million for trabecular). Reported values are the median with interquartile range (25th percentile-75th percentile). Morphometric parameters include: total image volume (TV), total bone volume (BV), ratio of bone volume to total volume (BV/TV), lacunar porosity (Lc.TV/BV), lacunar number (Lc.N), lacunar density (Lc.N/BV), local lacunar volume ( $\langle$ Lc.V $\rangle$ ), local lacunar surface area $(\langle$ Lc.S $\rangle)$, population lacunar volume ([Lc.V]), population lacunar surface area ([Lc.S]), population lacunar surface area to volume ratio ([Lc.S/Lc.V]), population lacunar stretch ([Lc.St]), population lacunar oblateness ([Lc.Ob]), population lacunar sphericity ([Lc.Sr]), population lacunar equancy ([Lc.Eq]), and population lacunar angle ([Lc. $\theta]$ ). Paired $t$-test performed for the normally distributed global and local parameters, $\left(^{*}\right)$ indicates $p<0.001$. Mann-Whitney $U$ test performed on population-based parameters and (*) indicates $p<0.001$.

\begin{tabular}{lll}
\hline Morphometric parameter & Trabecular & Cortical \\
\hline $\mathrm{TV}\left(\mathrm{mm}^{3}\right)$ & $15.7(15.1-15.8)$ & $15.7(15.6-15.9)$ \\
$\mathrm{BV}\left(\mathrm{mm}^{3}\right)$ & $2.11(1.54-2.46)^{*}$ & $7.68(6.33-9.70)$ \\
$\mathrm{BV} / \mathrm{TV}(\%)$ & $14.0(10.2-18.4)^{*}$ & $57.8(43.7-64.9)$ \\
Lc.TV/BV $(\%)$ & $0.30(0.22-0.38)^{*}$ & $0.58(0.51-0.65)$ \\
Lc.N $(1000)$ & $41.2(27.7-67.9)^{*}$ & $252(175-266)$ \\
Lc.N/BV $\left(1000 / \mathrm{mm}^{3}\right)$ & $16.6(14.1-18.7)^{*}$ & $26.4(23.7-29.3)$ \\
$\langle\mathrm{Lc} . \mathrm{V}\rangle\left(\mu \mathrm{m}^{3}\right)$ & $178(159-197)^{*}$ & $223(189-245)$ \\
$\langle\mathrm{Lc} . \mathrm{S}\rangle\left(\mu \mathrm{m}^{2}\right)$ & $194(178-211)^{*}$ & $233(216-248)$ \\
{$[\mathrm{Lc} . \mathrm{V}]\left(\mu \mathrm{m}^{3}\right)$} & $123(70.1-230)^{*}$ & $116(63.0-272)$ \\
{$[\mathrm{Lc} . \mathrm{S}]\left(\mu \mathrm{m}^{2}\right)$} & $161(109-245)^{*}$ & $164(105-290)$ \\
{$[\mathrm{Lc} . \mathrm{S}] /[\mathrm{Lc} . \mathrm{V}](1 / \mu \mathrm{m})$} & $1.30(1.05-1.56)^{*}$ & $1.41(1.06-1.66)$ \\
{$[\mathrm{Lc} . \mathrm{St}](1)$} & $0.62(0.53-0.69)^{*}$ & $0.61(0.51-0.69)$ \\
{$[\mathrm{Lc} . \mathrm{Ob}](1)$} & $-0.44(-0.67 \text { to }-0.13)^{*}$ & $-0.49(-0.71$ to -0.17$)$ \\
{$[\mathrm{Lc} . \mathrm{Sr}](1)$} & $0.76(0.70-0.81)^{*}$ & $0.73(0.66-0.79)$ \\
{$[\mathrm{Lc} . \mathrm{Eq}](1)$} & $0.29(0.19-0.45)^{*}$ & $0.31(0.19-0.47)$ \\
{$[\mathrm{Lc} . \theta]\left({ }^{\circ}\right)$} & $104(76.0-131)^{*}$ & $107(77.4-134)$ \\
\hline
\end{tabular}

landmark-based threshold to be applied to the image that accounted for sample-specific mineralization heterogeneities. Typically, a single threshold is chosen by the user via visual inspection and then applied to all samples in a study. At the tissue level this method is acceptable when recommended guidelines are carefully followed [49] and was how BV was calculated in this study. However, due to the wide variation of the TMD distributions between samples at the $1.2 \mu \mathrm{m}$ resolution, this singlethreshold method cannot be applied to all samples when imaging lacunae (Fig. S1). Therefore, our approach was to pragmatically locate a threshold that was intrinsically linked to the sample specific TMD distribution, compare the resulting lacunar identification with manual identification, and quantify the accuracy. Previous studies have tried similar individual threshold approaches, which are offset from a reference point of the TMD histogram such as the mean [50], but we found our specific images responded best to selecting the TMD histogram critical point as a landmark for segmentation (Fig. S1). In addition, the critical point is a clear and calculable landmark on every TMD histogram, which is less prone to error than an offset. This produced an adequate automated segmentation of lacunar structures as is depicted in Fig. 3. Alternative descriptors of the TMD distribution were investigated such as the width of the distribution but did not prove to be as effective as the first critical point (Fig. S2). It is important to note that the TMD distribution can be very heterogeneous around osteocyte lacunae and that adjustments to the algorithm would need to be made when investigating topics like osteocytic osteolysis which changes the mineral surrounding the lacunar structures. The $\mu$ CT50 machine was calibrated weekly with a multi-material phantom to minimize measurement errors with respect to scaling the raw signal attenuation to values of

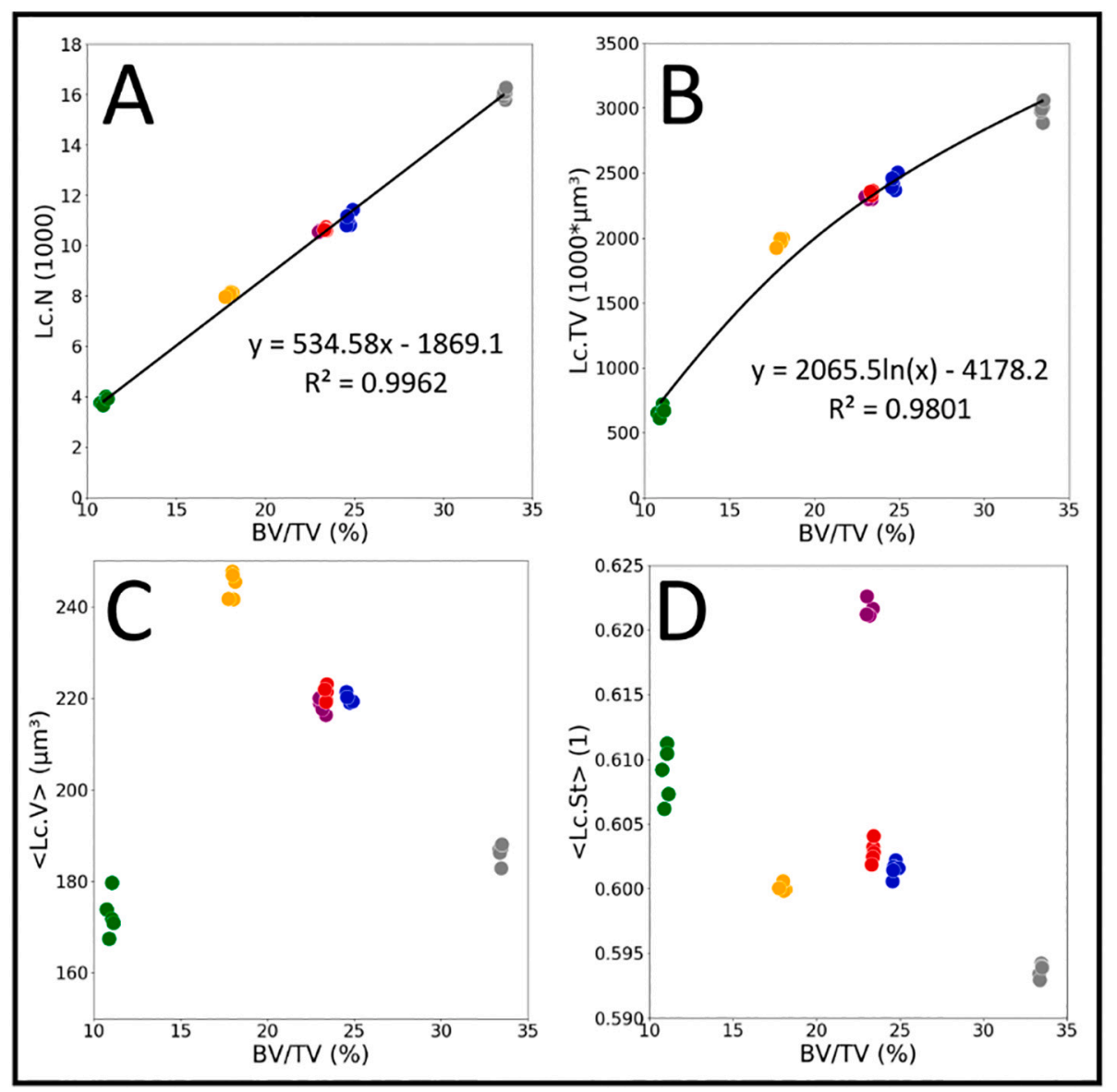

Fig. 5. Two-parameter plots that demonstrate the reproducibility of the imaging method. Each color represents an individual sample and each data point represents a specific measurement ( $\mathrm{n}=6$, with 5 repeated measurements). A) Lacunar number (Lc.N) vs. bone volume (BV/TV). B) Lacunar total volume (Lc.TV) vs. bone volume (BV/TV). C) Local lacunar volume (〈Lc.V $\rangle)$ vs. bone volume (BV/TV). D) Local lacunar stretch ( $\langle\mathrm{Lc} . \mathrm{St}\rangle)$ vs. bone volume (BV/TV). 

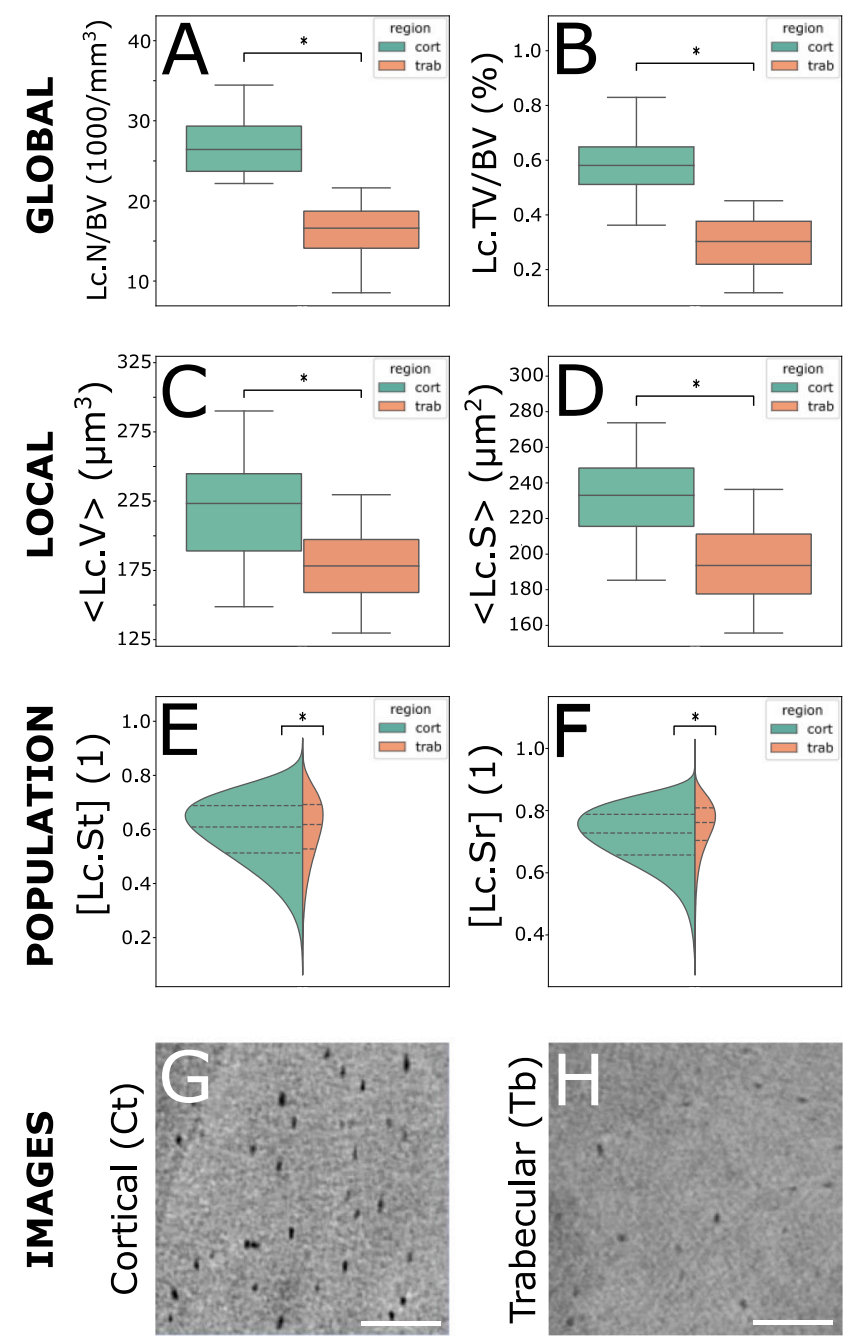

Fig. 6. Comparison of cortical and trabecular regions regarding lacunar morphometric parameters. Included in the analysis were 31 samples which comprised of 6.57 million cortical lacunae and 1.14 million trabecular lacunae. A) Lacunar density (Lc.N/BV). B) Lacunar porosity (Lc.TV/BV). C) Local lacunar volume $(\langle$ Lc.V $\rangle)$ normalized to sample. D) Local lacunar surface area $(\langle$ Lc.S $\rangle)$ normalized to sample. E) Population-based local lacunar stretch ([Lc. St]) not normalized to sample. F) Population-based local lacunar sphericity ([Lc.Sr]) not normalized to sample. G \& H) Micro-CT images from cortical and trabecular regions representing the median values from plot (A) respectively, scale bar $=100 \mu \mathrm{m}$. Paired $t$-test performed on normalized parameter plots (A-D) and (*) indicates $p<0.001$. Mann-Whitney $U$ test performed on population-based parameters $(\mathrm{E}-\mathrm{F})$ and $(*)$ indicates $p<0.001$.

hydroxyapatite for the TMD histograms.

The range of considered object volumes was also important for lacunar identification. This range varies substantially between studies and could be as narrow as $50-610 \mu \mathrm{m}^{3}$ or as wide as $175-2000 \mu \mathrm{m}^{3}$ $[18,21,42,43,51,52]$. Previous examinations of histological slides have estimated the human lacunae to be between $28 \mu^{3}$ and $1713 \mu \mathrm{m}^{3}$, yet lacunae observed below $50 \mu \mathrm{m}^{3}$ were only found in fracture callus [41]. After evaluating several different volume ranges and comparing both qualitatively with $2 \mathrm{D}$ and $3 \mathrm{D}$ images and quantitatively with accuracy measures such as TPR, FPR, and FNR, we determined a lower limit of 50 $\mu^{3}$ to be optimal for lacunar identification in human trabecular and cortical bone [53]. The upper limit was chosen to be $2000 \mu \mathrm{m}^{3}$ in line with a similar study [21]. This range has a large impact on the number of lacunae analyzed and is particularly sensitive on the lower limit. This problem is especially pronounced with respect to desktop micro-CT due to the limited photon count of its X-ray beam technology. Relative to studies conducted with synchrotron CT systems [18,42], the desktop micro-CT X-ray beam creates image projections with fewer photons, which increases the resulting noise, reduces the image quality, and makes visualization of small lacunae more difficult. A Gaussian filter with a low sigma value of 0.8 was applied so noise would be reduced while the borders of the lacunae would not be blurred beyond the recognition of a trained human observer. Several extremely elongated ring artifacts were observed in segmentations and were successfully removed by implementing an anti-ring reconstruction filter and applying a shape filter that removed objects with a Lc.St value greater than 0.85 [54].

We used ultra-high-resolution desktop micro-CT to image 7.71 million osteocyte lacunae across cortical and trabecular regions in 31 human iliac crest biopsies. We have observed that morphometric differences exist between lacunae in cortical and trabecular regions of bone, which has also been shown in previous studies [43,46]. Currently, only Akhter et al. [43] have reported sample matched trabecular and cortical lacunar morphometric parameters in human iliac crest biopsies. In contrast to their study, we report higher values of Lc.N/BV and Lc.TV/ $\mathrm{BV}$ in cortical bone when compared to trabecular bone. However, the narrow volume range they evaluate $(50-610 \mu \mathrm{m})$ and their analysis of less than $1 \%$ of the number of lacunae that we examine severely limits the range of variation that they could potentially consider. Additionally, we have proven our desktop micro-CT imaging technique to be accurate for lacunar identification, reproducible for repeated measures, and sensitive for lacunar parameters between anatomically distinct regions.

We evaluated not only global lacunar parameters related to tissue measures (Fig. 6A-B), but also local (Fig. 6C-D) and population-based (Fig. 6E-F) values. Population-based morphometry was not normalized and presents the reader with an undistorted perspective of the natural variation of certain morphometric indices across millions of lacunae (Fig. 6E-F). This further illustrates the method's sensitivity to biological differences that we see in Fig. 6A-D and also demonstrates the method's ability to capture the natural variation of lacunae in a largescale analysis.

Manual identification was used as our gold-standard for calculating accuracy, because registration between micro-CT images and typical morphological gold-standards like histology is extremely difficult. However, we were careful to create the best manual identification dataset possible for comparison. Cresswell et al. have used similar accuracy comparisons in previous studies and in fact, achieve similar rates of TP, FP, and FN to ours [50]. Interestingly, sample 3 in our accuracy calculation exhibited an inordinately low TPR and high FNR. This was due to a suboptimal selection of the sample's subregion near the bone surface, which made manual identification of lacunae slightly more difficult. Ultimately, this resulted in a slight reduction of the overall accuracy of lacunar identification. We did not investigate the impact this poor sample had on the resulting segmented morphometry as we only used these five samples to evaluate the accuracy of our method relative to lacunar identification. FN objects could only be visualized in $2 \mathrm{D}$ as the machine incorrectly did not segment these objects. However, the 3D position of the center voxel in the FN objects could still be evaluated in relation to TP and FP objects as this was the coordinate of the voxel chosen by the trained observer. Objects determined to be FP resembled noise structures and were either image artifacts or non-lacunar micropores.

We observed very low precision errors and high intraclass correlation coefficients with respect to our five consecutive measurements of six samples. These values were in the same range as in the study of Hemmatian et al. who investigated the reproducibility of desktop microCT for imaging murine lacunae [48]. The tight clustering of data points when creating two-parameter plots as seen in Fig. 5 further proves the reproducibility of the method. Fig. 5A and B both exhibit tight clustering within the measurements for each sample which is what we expect when comparing lacunar parameters with tissue values like BV/TV. Bone tissue volume is a quantity that micro-CT is excellent at measuring and 
hence we would expect it to be extremely reproducible. Physiologically speaking, we would also expect Lc.N and Lc.TV to increase with increasing total bone volume, which explains the strong correlation, further adds credibility to the imaging modality, and even positions the two lacunar parameters as potential biomarker candidates. Previous research has demonstrated osteocytic osteolysis plays a role in bone health, disease, and mechanisms of medication response; this also poises the lacunar microarchitecture as a potential biomarker from the perspective of osteocyte-controlled bone remodeling [55]. Furthermore, a variety of chemically based bone biomarkers, such as serum CTX and $\mathrm{P} 1 \mathrm{NP}$, currently exist for the clinical assessment of osteoporosis/bone remodeling and lacunar parameters like Lc.N and Lc.TV could potentially be used in conjunction with those recently outlined by Kuo et al. for investigation of disease mechanisms [56]. Yet in Fig. 5C and D, we note that the values of each sample are slightly less clustered in comparison with Fig. 5A and B and are not correlated. More specifically, we note that BV/TV remains very consistent but the $\langle$ Lc.V $\rangle$ and $\langle$ Lc.St $\rangle$ varies. Smaller lacunar objects are difficult to mesh and more prone to measurement errors due to the comparatively large voxel size. Consequently, these small objects have a relatively coarser mesh. Both $\langle\mathrm{Lc} . \mathrm{V}\rangle$ and $\langle$ Lc.St $\rangle$ are dependent on the object mesh which would explain the reduced reproducibility in comparison to Lc.N and Lc.TV. Hemmatian et al. also found lacunar measures such as $\langle\mathrm{Lc} . \mathrm{V}\rangle$ to be less reproducible than tissue measures such as BV/TV [48]. Consequently, $\langle$ Lc.V $\rangle$ and $\langle$ Lc. St) do not appear to be good biomarker candidates.

Previous studies have demonstrated that lacunar morphometric parameters differ between trabecular and cortical regions of bone $[43,46,57]$. We used this observation to evaluate the sensitivity of our method to biological differences by the ability to differentiate lacunae between anatomically distinct regions. This was based upon the analysis performed by Nebuloni et al. regarding the sensitivity of micro-CT to detect biological differences relative to vascular imaging [47]. We report significant differences between global, local, and populationbased parameters including Lc.TV/BV, Lc.N/BV, $\langle$ Lc.V $\rangle,\langle$ Lc.S $\rangle$, [Lc.St], and [Lc.Sr] as seen in Table 4 and Fig. 6A-F. Furthermore, Fig. 6G-H provide a visual confirmation of the difference that we report in Fig. 6A. Not only do we see that lacunar density is lower in trabecular regions, but also the lacunae themselves look to be slightly smaller relative to the cortical regions. This would indicate that lacunae in trabecular regions also consist of lower total porosity (Lc.TV/BV), volume ( $\langle$ Lc.V $\rangle)$, and surface area ( $\langle$ Lc.S $\rangle)$. These visual differences further support our claim that the method is sensitive to previously studied regional differences and reflects the statistically significant differences that we report in Fig. 6A-D. Kegelman et al. found similar lacunar morphometries in a recent study; however, overall their lacunae were slightly smaller, likely because they were of murine origin [57].

While we were able to evaluate the accuracy of lacunar identification between human and machine counting, we did not address the accuracy of the segmentation of the lacunar structures as other studies have done [48]. This is a limitation of our study and would be interesting to quantify. However, because lacunar segmentation is resolution dependent and Hemmatian et al. demonstrated that lacunar geometry is correlated, but not accurate, between desktop micro-CT and CLSM, we believe lacunar identification accuracy is more meaningful [48]. Additionally, the lacunar data were very sensitive to the selection of the lower volumetric bound. This was difficult to select since volumetric data from previous studies regarding the distinction between a lacuna and a micropore is limited. Furthermore, we must acknowledge the fact that partial volume effects generate error with respect to the segmented lacunar structures. Considering many lacunae have a diameter of roughly $10 \mu \mathrm{m}$, these partial volume effects are accentuated at the 1.2 $\mu \mathrm{m}$ voxel resolution. Finally, the precision study required weeks of scanning time and consequently was only performed on trabecular bone.

\section{Conclusion}

We present a new high-throughput method that is reproducible and sensitive to assess osteocyte lacunar morphometry in human bone samples. We use ultra-high-resolution desktop micro-CT, an individualized histogram-based segmentation procedure, and a custom evaluation algorithm to calculate global and local morphometric parameters of 7.71 million lacunae in two distinct regions of 31 human iliac crest bone samples, revealing two potential biomarkers. The validation of our method demonstrates accurate lacunar identification, reproducibility of repeated measurements, and sensitivity between anatomically distinct regions. Therefore, our new image acquisition and evaluation methodologies expand the number of investigable hypotheses surrounding osteocyte lacunae, while simultaneously employing a widely accessible and mature imaging technology — desktop micro-CT.

\section{CRediT authorship contribution statement}

Elliott Goff: Conceptualization, Methodology, Software, Validation, Formal analysis, Investigation, Data curation, Writing - original draft, Writing - review \& editing, Visualization. Federica Buccino: Software, Validation, Methodology, Investigation. Chiara Bregoli: Software, Validation, Methodology, Investigation. Jonathan P. McKinley: Software, Validation, Methodology, Investigation. Basil Aeppli: Software, Validation, Methodology, Investigation. Robert R. Recker: Resources. Elizabeth Shane: Resources, Writing - review \& editing. Adi Cohen: Resources, Writing - review \& editing. Gisela Kuhn: Supervision, Writing - review \& editing. Ralph Müller: Supervision, Resources, Project administration, Writing - review \& editing.

\section{Acknowledgements}

The authors would like to thank the Joint Scoliosis Research Center of The Chinese University of Hong Kong and Nanjing University, Hong Kong \& Nanjing, China for their joint efforts on providing and processing the bone biopsies used for beam energy optimization. We thank Lucid AG for assisting with the resolution dependency study using XamFlow. We would also like to thank Peter Schwilch for assisting with biopsy machining and Dr. Patrik Christen for his mentoring.

\section{Appendix A. Supplementary data}

Supplementary data to this article can be found online at https://doi. org/10.1016/j.bone.2021.116094.

\section{References}

[1] J. Wolff, Das Gesetz der Transformation der Knochen, DMW-Deutsche Medizinische Wochenschrift 19 (47) (1892) 1222-1224.

[2] W. Roux, Der Kampf der Theile im Organismus: ein Beitrag zur Vervollständigung der mechanischen Zweckmässigkeitslehre, W. Engelmann, 1881.

[3] L.F. Bonewald, The amazing osteocyte, J. Bone Miner. Res. 26 (2) (2011) 229-238.

[4] P.R. Buenzli, N.A. Sims, Quantifying the osteocyte network in the human skeleton, Bone 75 (79) (2015) 144-150.

[5] J. Gluhak-Heinrich, L. Ye, L.F. Bonewald, J.Q. Feng, M. MacDougall, S.E. Harris, D. Pavlin, Mechanical loading stimulates dentin matrix protein 1 (DMP1) expression in osteocytes in vivo, J. Bone Miner. Res. 18 (5) (2003) 807-817.

[6] A.G. Robling, P.J. Niziolek, L.A. Baldridge, K.W. Condon, M.R. Allen, I. Alam, S. M. Mantila, J. Gluhak-Heinrich, T.M. Bellido, S.E. Harris, C.H. Turner, Mechanical stimulation of bone in vivo reduces osteocyte expression of Sost/sclerostin, J. Biol. Chem. 283 (9) (2008) 5866-5875.

[7] T.M. Skerry, L. Bitensky, J. Chayen, L.E. Lanyon, Early strain-related changes in enzyme activity in osteocytes following bone loading in vivo, J. Bone Miner. Res. 4 (5) (1989) 783-788.

[8] L. Lanyon, Osteocytes, strain detection, bone modeling and remodeling, Calcif. Tissue Int. 53 (1) (1993) S102-S107.

[9] F.A. Schulte, D. Ruffoni, F.M. Lambers, D. Christen, D.J. Webster, G. Kuhn, R. Muller, Local mechanical stimuli regulate bone formation and resorption in mice at the tissue level, PLoS One 8 (4) (2013), e62172.

[10] R. Huiskes, R. Ruimerman, G.H. van Lenthe, J.D. Janssen, Effects of mechanical forces on maintenance and adaptation of form in trabecular bone, Nature 405 (6787) (2000) 704-706. 
[11] S. Hirose, M. Li, T. Kojima, P. Henrique, L. de Freitas, S. Ubaidus, K. Oda, C. Saito, N. Amizuka, A histological assessment on the distribution of the osteocytic lacunar canalicular system using silver staining, J. Bone Miner. Metab. 25 (6) (2007) 374-382.

[12] C. Palumbo, A 3-dimensional ultrastructural-study of osteoid-osteocytes in the tibia of chick-embryos, Cell Tissue Res. 246 (1) (1986) 125-131.

[13] G. Marotti, F. Remaggi, D. Zaffe, Quantitative investigation on osteocyte canaliculi in human compact and spongy bone, Bone 6 (5) (1985) 335-337.

[14] D.J. Kubek, V.H. Gattone, M.R. Allen, Methodological assessment of acid-etching for visualizing the osteocyte lacunar-canalicular networks using scanning electron microscopy, Microsc. Res. Tech. 73 (3) (2010) 182-186.

[15] C.M. Heveran, A. Rauff, K.B. King, R.D. Carpenter, V.L. Ferguson, A new opensource tool for measuring 3D osteocyte lacunar geometries from confocal laser scanning microscopy reveals age-related changes to lacunar size and shape in cortical mouse bone, Bone 110 (2018) 115-127.

[16] X.H. Lai, C. Price, S. Modla, W.R. Thompson, J. Caplan, C.B. Kirn-Safran, L. Y. Wang, The dependences of osteocyte network on bone compartment, age, and disease, Bone Res. 3 (2015) 11

[17] F. Repp, P. Kollmannsberger, A. Roschger, M. Kerschnitzki, A. Berzlanovich, G. M. Gruber, P. Roschger, W. Wagermaier, R. Weinkamer, Spatial heterogeneity in the canalicular density of the osteocyte network in human osteons, Bone Rep. 6 (2017) 101-108.

[18] K.S. Mader, P. Schneider, R. Muller, M. Stampanoni, A quantitative framework for the 3D characterization of the osteocyte lacunar system, Bone 57 (1) (2013) $142-154$.

[19] M.G. Ascenzi, J. Gill, A. Lomovtsev, Orientation of collagen at the osteocyte lacunae in human secondary osteons, J. Biomech. 41 (16) (2008) 3426-3435.

[20] H.M. Britz, Y. Carter, J. Jokihaara, O.V. Leppanen, T.L.N. Jarvinen, G. Belev, D.M. L. Cooper, Prolonged unloading in growing rats reduces cortical osteocyte lacunar density and volume in the distal tibia, Bone 51 (5) (2012) 913-919.

[21] Y. Carter, C.D.L. Thomas, J.G. Clement, A.G. Peele, K. Hannah, D.M.L. Cooper, Variation in osteocyte lacunar morphology and density in the human femur - a synchrotron radiation micro-CT study, Bone 52 (1) (2013) 126-132.

[22] P. Schneider, M. Meier, R. Wepf, R. Muller, Towards quantitative 3D imaging of the osteocyte lacuno-canalicular network, Bone 47 (5) (2010) 848-858.

[23] M. Langer, A. Pacureanu, H. Suhonen, Q. Grimal, P. Cloetens, F. Peyrin, X-ray phase nanotomography resolves the 3D human bone ultrastructure, PLoS One 7 (8) (2012) 7 .

[24] M. Kerschnitzki, W. Wagermaier, P. Roschger, J. Seto, R. Shahar, G.N. Duda, S. Mundlos, P. Fratzl, The organization of the osteocyte network mirrors the extracellular matrix orientation in bone, J. Struct. Biol. 173 (2) (2011) 303-311.

[25] L.P. Bakalova, C.M. Andreasen, J.S. Thomsen, A. Bruel, E.M. Hauge, B.J. Kiil, J. M. Delaisse, T.L. Andersen, M.E Kersh, Intracortical bone mechanics are related to pore morphology and remodeling in human bone, J. Bone Miner. Res. 33 (12) (2018) 2177-2185.

[26] M.L. Bouxsein, S.K. Boyd, B.A. Christiansen, R.E. Guldberg, K.J. Jepsen, R. Muller, Guidelines for assessment of bone microstructure in rodents using micro-computed tomography, J. Bone Miner. Res. 25 (7) (2010) 1468-1486.

[27] T. Hildebrand, A. Laib, R. Muller, J. Dequeker, P. Ruegsegger, Direct threedimensional morphometric analysis of human cancellous bone: microstructural data from spine, femur, iliac crest, and calcaneus, J. Bone Miner. Res. 14 (7) (1999) 1167-1174.

[28] R. Muller, H. Van Campenhout, B. Van Damme, G. Van Der Perre, J. Dequeker, T. Hildebrand, P. Ruegsegger, Morphometric analysis of human bone biopsies: a quantitative structural comparison of histological sections and micro-computed tomography, Bone 23 (1) (1998) 59-66.

[29] P. Schneider, M. Stauber, R. Voide, M. Stampanoni, L.R. Donahue, R. Muller, Ultrastructural properties in cortical bone vary greatly in two inbred strains of mice as assessed by synchrotron light based micro- and nano-CT, J. Bone Miner. Res. 22 (10) (2007) 1557-1570.

[30] Y. Sugawara, H. Kamioka, T. Honjo, K. Tezuka, T. Takano-Yamamoto, Threedimensional reconstruction of chick calvarial osteocytes and their cell processes using confocal microscopy, Bone 36 (5) (2005) 877-883.

[31] R.P. van Hove, P.A. Nolte, A. Vatsa, C.M. Semeins, P.L. Salmon, T.H. Smit, J. KleinNulend, Osteocyte morphology in human tibiae of different bone pathologies with different bone mineral density — is there a role for mechanosensing? Bone 45 (2) (2009) 321-329.

[32] A. Vatsa, R.G. Breuls, C.M. Semeins, P.L. Salmon, T.H. Smit, J. Klein-Nulend, Osteocyte morphology in fibula and calvaria - is there a role for mechanosensing? Bone 43 (3) (2008) 452-458.

[33] S. Suniaga, T. Rolvien, A. vom Scheidt, I.A.K. Fiedler, H.A. Bale, A. Huysseune, P. E. Witten, M. Amling, B. Busse, Increased mechanical loading through controlled swimming exercise induces bone formation and mineralization in adult zebrafish, Sci. Rep. 8 (2018) 13.

[34] B. Ay, K. Parolia, R.S. Liddell, Y.S. Qiu, G. Grasselli, D.M.L. Cooper, J.E. Davies, Hyperglycemia compromises rat cortical bone by increasing osteocyte lacunar density and decreasing vascular canal volume, Commun. Biol. 3 (1) (2020) 9.
[35] C.-C. Glüer, G. Blake, Y. Lu, B. Blunt, M. Jergas, H. Genant, Accurate assessment of precision errors: how to measure the reproducibility of bone densitometry techniques, Osteoporos. Int. 5 (4) (1995) 262-270.

[36] A. Cohen, D.W. Dempster, R.R. Recker, E.M. Stein, J.M. Lappe, H. Zhou, A.J. Wirth, G.H. van Lenthe, T. Kohler, A. Zwahlen, R. Muller, C.J. Rosen, S. Cremers, T. L. Nickolas, D.J. McMahon, H. Rogers, R.B. Staron, J. LeMaster, E. Shane, Abnormal bone microarchitecture and evidence of osteoblast dysfunction in premenopausal women with idiopathic osteoporosis, J. Clin. Endocrinol. Metab. 96 (10) (2011) 3095-3105.

[37] A. Cohen, R.R. Recker, J. Lappe, D.W. Dempster, S. Cremers, D.J. McMahon, E. M. Stein, J. Fleischer, C.J. Rosen, H. Rogers, R.B. Staron, J. Lemaster, E. Shane, Premenopausal women with idiopathic low-trauma fractures and/or low bone mineral density, Osteoporos. Int. 23 (1) (2012) 171-182.

[38] H.X. Chen, J.J. Zhang, Y.J. Wang, K.Y. Cheuk, A.L.H. Hung, T.P. Lam, Y. Qiu, J. Q. Feng, W.Y.W. Lee, J.C.Y. Cheng, Abnormal lacuno-canalicular network and negative correlation between serum osteocalcin and Cobb angle indicate abnormal osteocyte function in adolescent idiopathic scoliosis, Faseb J. 33 (12) (2019) 13882-13892.

[39] M. Mashiatulla, R.D. Ross, D.R. Sumner, Validation of cortical bone mineral density distribution using micro-computed tomography, Bone 99 (2017) 53-61.

[40] M. Firbank, A. Coulthard, R. Harrison, E. Williams, A comparison of two methods for measuring the signal to noise ratio on MR images, Phys. Med. Biol. 44 (12) (1999) N261.

[41] B.R. McCreadie, S.J. Hollister, M.B. Schaffler, S.A. Goldstein, Osteocyte lacuna size and shape in women with and without osteoporotic fracture, J. Biomech. 37 (4) (2004) 563-572.

[42] F.L. Bach-Gansmo, A. Bruel, M.V. Jensen, E.N. Ebbesen, H. Birkedal, J.S. Thomsen, Osteocyte lacunar properties and cortical microstructure in human iliac crest as a function of age and sex, Bone 91 (2016) 11-19.

[43] M.P. Akhter, D. Kimmel, J. Lappe, R. Recker, Effect of macroanatomic bone type and estrogen loss on osteocyte lacunar properties in healthy adult women, Calcif. Tissue Int. (2017) 1-12.

[44] M. Stauber, R. Muller, Volumetric spatial decomposition of trabecular bone into rods and plates — a new method for local bone morphometry, Bone 38 (4) (2006) 475-484.

[45] A. Carriero, M. Doube, M. Vogt, B. Busse, J. Zustin, A. Levchuk, P. Schneider, R. Muller, S.J. Shefelbine, Altered lacunar and vascular porosity in osteogenesis imperfecta mouse bone as revealed by synchrotron tomography contributes to bone fragility, Bone 61 (2014) 116-124.

[46] P. Milovanovic, B. Busse, Inter-site variability of the human osteocyte lacunar network: implications for bone quality, Curr. Osteoporos. Rep. 17 (2019) 105-115.

[47] L. Nebuloni, G.A. Kuhn, J. Vogel, R. Muller, A novel in vivo vascular imaging approach for hierarchical quantification of vasculature using contrast enhanced micro-computed tomography, PLoS One 9 (1) (2014) 10.

[48] H. Hemmatian, M.R. Laurent, S. Ghazanfari, D. Vanderschueren, A.D. Bakker, J. Klein-Nulend, G.H. van Lenthe, Accuracy and reproducibility of mouse cortical bone microporosity as quantified by desktop microcomputed tomography, PLoS One 12 (8) (2017).

[49] M.L. Bouxsein, S.K. Boyd, B.A. Christiansen, R.E. Guldberg, K.J. Jepsen, R. Muller, Guidelines for assessment of bone microstructure in rodents using micro-computed tomography, J. Bone Miner. Res. 25 (7) (2010) 1468-1486.

[50] E.N. Cresswell, T.M. Nguyen, M.W. Horsfield, A.J. Alepuz, T.A. Metzger, G. L. Niebur, C.J. Hernandez, Mechanically induced bone formation is not sensitive to local osteocyte density in rat vertebral cancellous bone, J. Orthop. Res. 36 (2) (2017) 672-681.

[51] A. Levchuk, P. Schneider, M. Meier, P. Vogel, F. Donaldson, R. Muller, An automated step-wise micro-compression device for 3D dynamic image-guided failure assessment of bone tissue on a microstructural level using time-lapsed tomography, Front. Mater. 5 (2018) 14.

[52] S.M. Tommasini, A. Trinward, A.S. Acerbo, F. De Carlo, L.M. Miller, S. Judex, Changes in intracortical microporosities induced by pharmaceutical treatment of osteoporosis as detected by high resolution micro-CT, Bone 50 (3) (2012) 596-604.

[53] B. Aeppli, R. Müller, E. Goff, A novel shape filter for lacunar imaging in human bone using ultra-high-resolution desktop micro-CT, in: M.O.P. Nithiarasu, M. Oshima (Eds.), 6th International Conference on Computational and Mathematical Biomedical Engineering (CMBE 2019), Sendai City, Japan, 2019, pp. 651-654.

[54] J. Sijbers, A. Postnov, Reduction of ring artefacts in high resolution micro-CT reconstructions, Phys. Med. Biol. 49 (14) (2004) N247-N253.

[55] E. Tsourdi, K. Jahn, M. Rauner, B. Busse, L.F. Bonewald, Physiological and pathological osteocytic osteolysis, J. Musculoskelet. Neuronal Interact. 18 (3) (2018) 292-303.

[56] T.R. Kuo, C.H. Chen, Bone biomarker for the clinical assessment of osteoporosis: recent developments and future perspectives, Biomark. Res. 5 (2017) 18.

[57] C.D. Kegelman, J.C. Coulombe, K.M. Jordan, D.J. Horan, L. Qin, A.G. Robling, V. L. Ferguson, T.M. Bellido, J.D. Boerckel, YAP and TAZ mediate osteocyte perilacunar/canalicular remodeling, J. Bone Miner. Res. 35 (1) (2020) 196-210. 